\title{
Influence of restoration adjustments on prefrontal blood flow
}

\author{
A simplified NIRS preliminary study \\ Kenichi Sasaguri · Takero Otsuka · Hiroyuki Tsunashima - Tateshi Shimazaki · Kin-Ya Kubo • \\ Minoru Onozuka
}

Received: 24 February 2015 / Accepted: 3 March 2015 / Published online: 27 March 2015

(C) Springer-Verlag Wien 2015

\begin{abstract}
Objective The aim of this study was to examine, after setting several restorations, the influence of adjusted occlusal interference during gum chewing on blood flow in the prefrontal area as determined using near-infrared spectroscopy.

Material and methods The physiological rate was assessed using a visual analog scale (VAS) questionnaire. We selected 16 patients who desired prosthetic restorative treatment on the lateral dentition, and eight healthy volunteers. Subjects were divided into three eight-person groups. One group received restorations on the premolar area (PA), another group received restorations on the molar area (MA), and the control group (CT) received no prosthetic restorations. The spectroscope was fastened to the frontal region of the head after placement of the final restoration, but before adjustment.
\end{abstract}

Kenichi Sasaguri and Takero Otsuka contributed equally to this work.

T. Otsuka, D.D.S, Ph.D $(\bowtie) \cdot$ K. Sasaguri · T. Shimazaki Division of Orthodontics, Department of Oral Science, Kanagawa Dental University Graduate School, 82 Inaoka-cho,

238-8580 Yokosuka, Kanagawa, Japan

e-mail: otsuka@kdu.ac.jp

\section{H. Tsunashima}

Division of Brain functions and Neuroscience, Department of Oral

Science, Kanagawa Dental University Graduate School,

Yokosuka, Kanagawa, Japan

K.-Y. Kubo

Seijoh University Graduate School of Health Care Studies,

Tokai, Aichi, Japan

M. Onozuka

Department of Medical Science, Nippon Sport Science University, Kumashida-cho, Aoba-ku,

Yokohama, Kanagawa, Japan
Results Pre-adjustment (first gum chewing for CT) blood flow in the prefrontal cortex was measured during gum chewing. Blood flow was again measured during gum chewing after the restoration (second gum chewing for CT) had been adjusted in accordance with the subjective assessment of the patient while wearing the device. The VAS provided quantification of comfort during gum chewing before and after restoration adjustment. For the PA and MA groups, adjusting restorations decreased discomfort significantly during gum chewing. Moreover, in the MA group, prefrontal blood flow was significantly reduced, and blood flow correlated with discomfort.

Conclusions Activation of the prefrontal area may provide an objective criterion for judging the functionality of occlusion after prosthetic occlusal reconstruction and/or orthodontics.

Keywords Chewing - Occlusal adjustment - Prefrontal cortex $\cdot$ Restorations $\cdot$ Stress

\section{Introduction}

Various prosthetic restorations are fabricated and fitted in clinical dentistry with the aim of restoring occlusal function. Once the restoration is attached, however, final occlusal adjustments are typically made based on subjective feedback from the patient, rather than on the basis of objective evidence gathered by dental technicians.

Some studies have reported that many patients with malocclusion complaints experience comorbid mental stress and somatic symptoms [1-3]. Additional studies have suggested that malocclusion may be linked to higher brain function [4-6]. An experimental animal study induced occlusal disharmony in rats by attaching metal wires to the biting surfaces of both maxillary molar teeth with resin cement to artificially raise the vertical dimension of occlusion (VDO). This experimental 
occlusal interference in rats caused chronic stress and altered long-term potentiation (LTP) of the hippocampus by triggering significant increases in plasma concentrations of the stress hormones corticosterone and norepinephrine [7]. Another study investigated the effects of experimental malocclusion in humans using functional magnetic resonance imaging (fMRI). That study found that changes in malocclusion were promptly recognized by the upper central nervous system as afferent information, subsequently activating the amygdala, anterior cingulate cortex, insula, and prefrontal cortex (PFC), all of which are involved in emotional processing and regulation. This resulted in feelings of discomfort as indicated by visual analog scale (VAS) scores [5]. Another fMRI study revealed that the severity of malocclusion correlates to PFC activity, which is involved in emotion [6]. These findings suggest that, if not performed properly, irreversible occlusal treatment (such as prosthodontic and orthodontic treatments) may act on higher brain function to produce chronic stress and other adverse somatic effects.

The PFC has been reported not only to be involved in various cognitive functions and emotions, but also to play important roles in controlling the autonomic nervous system and endocrine system involved in stress responses [8]. Neuroimaging studies have revealed that the medial prefrontal cortex (mPFC) is involved in emotional processing of stress $[9,10]$.

Current methods used to measure human brain function in medical and clinic research include positron emission tomography (PET), magnetoencephalography (MEG), fMRI, and near-infrared spectroscopy (NIRS). These neuroimaging techniques have also been used in the field of dentistry to study the effects of various oral functions on the brain $[4,5,11-20]$. However, the results of this research have yet to be translated into clinical dental applications, since the aforementioned systems are often expensive and difficult to operate, while fMRI and MEG require the patient's head to be secured and the patient's posture to be limited. NIRS, on the other hand, is a noninvasive technique that can be used to measure brain activity via changes in cerebral blood flow (mainly in the cerebral cortex) by monitoring hemoglobin concentrations using near-infrared light [21]. NIRS devices can also be miniaturized to suit specific purposes, allowing brain activity to be assessed with the subject in any body position and without having to remain perfectly still.

The present study used a compact NIRS model (Optical Encephalography Spectratech OEG-16; Spectratech, Kanagawa, Japan) with the aim of collecting data for development of a system to provide straightforward, objective feedback on occlusal treatment and brain function that can be used during treatment in clinical dental settings. Changes in mPFC activity were measured by having patients chew gum before (i.e., baseline) and after final occlusal adjustment of the prosthetic restoration while wearing the OEG-16 head module. We also attempted to identify areas of brain activity representing useful, objective occlusal indicators by examining correlations with subjective assessment of masticatory function using a VAS.

\section{Material and methods}

\section{Subjects}

Subjects comprised 16 patients attending a general dental clinic (seven 7 men, mean age, $52.4 \pm 20.1$ years, range, 32-85 years; nine women, mean age, $42.7 \pm 12.3$ years, range, $24-70$ years; total mean age, $46.9 \pm 16.3$ years, range, 24-85 years) who were scheduled to undergo final fitting of a prosthetic appliance to a molar or premolar tooth and eight healthy volunteers (four men, mean age, 31.75 \pm 2.36 years, range, 30-35 years; four women, mean age, $28 \pm 1.2$ years, range, $27-29$ years; total mean age, $29.88 \pm 2.6$ years, range, $27-35$ years) without previous history of orthodontic or prosthodontic treatment. Written informed consent was obtained from all patients and volunteers after explaining the details of the study, including the aims, contents, and potential risks. Subjects were divided into a molar group $(n=8)$, a premolar group $(n=8)$, and a control group $(n=8)$ according to the restoration site. Table 1 shows patient sex and age, in addition to the site and type of restoration. This study was conducted with the approval of the ethics review board at Kanagawa Dental University (Approval no. 158).

\section{Measurement of cerebral blood flow}

The aim of the present study was to identify a method by which the quality of clinical dental treatment can be determined by focusing on brain activity in the presence or absence of an experience. Therefore, the OEG16 unit was chosen from among various NIRS devices based on its ability to selectively measure PFC activity, in addition to the compact design and simple operation (Fig. 1a). To measure changes in cerebral blood flow in the PFC, we applied probes using the International 10/20 system commonly used in electroencephalography [22]. The sagittal line was determined by drawing a line from the nasion to the inion. Next, a point $10 \%$ superior to the nasion on this line was taken as the front polar position (Fpz). A line passing through the nasion, pre-auricular point, and inion was then drawn as a horizontal reference line. The OEG-16 head unit was attached so that the bottom lines of the probe were parallel to the horizontal reference line, and the probe of the center of the bottom line was located at the Fpz.

\section{Study design}

After final fitting of the restoration but before adjustment, the OEG-16 unit was attached and the patient was instructed to remain still in a dental chair with eyes closed 
Table 1 Sex, age, and restoration type ([.e., inlay with vital pulp or full cast crown (FCC) with dead pulp] for each patient, according to restoration site (i.e., molar or premolar)

\begin{tabular}{|c|c|c|c|c|c|c|c|}
\hline Molar & Sex & Age & $\begin{array}{l}\text { Restration site/ } \\
\text { type }\end{array}$ & Premolar & Sex & Age & $\begin{array}{l}\text { Restration site/ } \\
\text { type }\end{array}$ \\
\hline M1 & Male & 32 & $\begin{array}{l}\text { Upper right } 7 / \\
\text { inlay }\end{array}$ & P1 & Male & 75 & Upper left 4/FCC \\
\hline M2 & Male & 85 & $\begin{array}{l}\text { Lower right } 7 / \\
\text { FCC }\end{array}$ & P2 & Male & 38 & Upper left 5/inlay \\
\hline M3 & Male & 46 & $\begin{array}{l}\text { Lower right 6/ } \\
\text { FCC }\end{array}$ & P3 & Male & 52 & Lower left 5/FCC \\
\hline M4 & Male & 39 & Lower left 7/FCC & P4 & Female & 24 & $\begin{array}{l}\text { Upper right 5/ } \\
\text { FCC }\end{array}$ \\
\hline M5 & Female & 40 & $\begin{array}{l}\text { Upper right 6/ } \\
\text { inlay }\end{array}$ & P5 & Female & 34 & Lower left 5/inlay \\
\hline M6 & Female & 41 & $\begin{array}{l}\text { Lower right } 7 / \\
\text { FCC }\end{array}$ & P6 & Female & 70 & Lower left 5/inlay \\
\hline M7 & Female & 41 & Lower left 6/FCC & P7 & Female & 44 & Upper left 5/FCC \\
\hline M8 & Female & 47 & $\begin{array}{l}\text { Lower right } 7 / \\
\text { inlay }\end{array}$ & P8 & Female & 43 & Upper left 5/FCC \\
\hline
\end{tabular}

Fig. 1 Attachment of the nearinfrared spectroscopy system (OEG-16). a Optical encephalography was performed using the OEG-16. b The compact design and simple attachment and removal of the device enabled simple measurement even during treatment. c This block design task was repeated three times each before and after adjustment of the restoration. Patients were instructed to remain still for the first $30 \mathrm{~s}$ and to chew a piece of gum for the next $20 \mathrm{~s}$. d Patients were also asked to assess the level of discomfort when chewing a piece of gum before and after adjustment by selecting a score of $0-10$ on a visual analog scale (VAS)

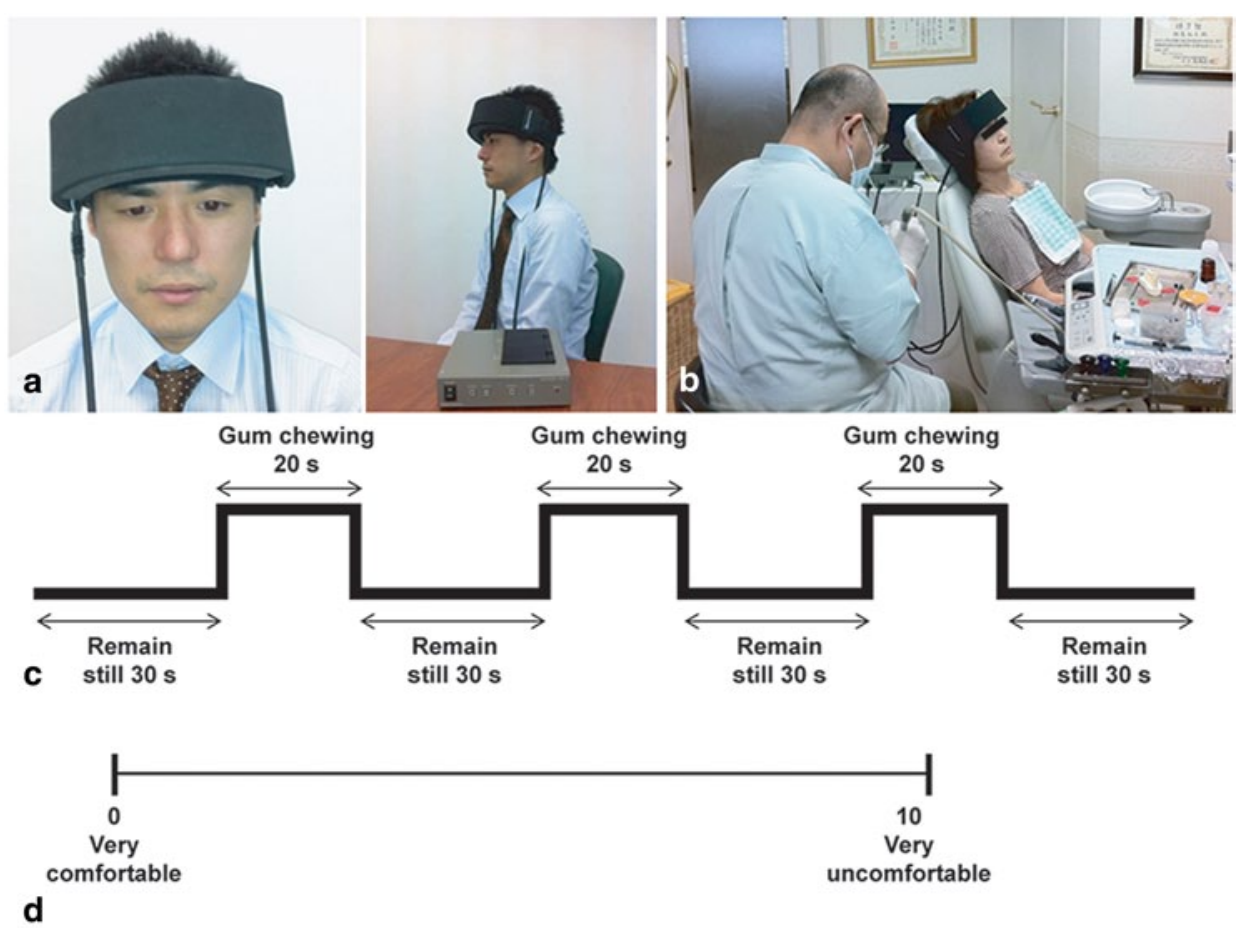

(Fig. 1b). Baseline preadjustment measurement consisted of a 30 -s rest phase during which the patient was instructed not to move, followed by a 20 -s chewing phase in which the patient was required to chew a piece of gum ( $1.5 \mathrm{~g}$, Free Zone; Lotte, Tokyo, Japan) in order to quantify mastication-induced blood flow in the PFC. The patient was required to chew at about $1 \mathrm{~Hz}$, representing a normal adult chewing rate as determined by reference to a previous study [23]. Finally, after stopping gum chewing, another 30-s rest phase was provided. This measurement sequence was repeated a total of three times (Fig. 1c). After the restoration had been adjusted in accordance with the subjective assessment of the patient, the resto- ration was set with resin-modified glass-ionomer cement (GC Fuji Luting, Tokyo, Japan). Postadjustment measurement was then performed with the OEG-16 unit attached based on the sequence described above. The mean ( \pm standard deviation) interval between pre- and postadjustment measurements was $17.4 \pm 6.2 \mathrm{~min}$. Exactly the same experimental protocol was applied for the control group except no prosthetic restoration was performed. Instead of adjustment, control group subjects remained seated in the dental chair for the mean adjustment time (17.4 min) between the first gum-chewing measurement (as surrogate for preadjustment) and the second gumchewing measurement (as surrogate for postadjustment). 


\section{Subjective assessment}

Subjective patient assessment of mastication before and after occlusal adjustment was achieved by assigning a VAS score of $0-10$, with 0 indicating "very comfortable" and 10 denoting "very uncomfortable" (Fig. 1d). The resulting data were subjected to one-way or two-way repeated measure analysis of variance (ANOVA) using grouping as the key variable when appropriate, and multiple comparison testing using Fisher's protected leastsignificant difference (PLSD). The significance level was set at $5 \%$.

\section{Analysis of correlation between brain activity data and VAS scores}

Oxygenated hemoglobin (oxy-Hb) was used as an indicator of brain activity owing to its strong correlation with neural activity $[24,25]$. Oxy-Hb values recorded in all NIRS channels during chewing phases before and after occlusal adjustment were averaged and subjected to one- or two-way repeated-measures ANOVA using grouping as the key variable when appropriate, and multiple comparison testing with Fisher's PLSD $(<5 \%$ significance level). The correlation between VAS score and brain activity was then statistically analyzed using Pearson's product moment correlation coefficient ( $r$; $<5 \%$ significance level). All statistical analyses were performed using the Statcel statistical package (Statcel 3; OMS, Tokorozawa, Japan).

\section{Results}

Figure 2 shows results for patient M3 before and after adjustment of the restoration. Data from all channels were included in the study results, except for channels 1-3 and 14-16, which were deemed to show interference in the NIRS signal due to temporalis muscle activity from gum chewing. Patients were divided into the molar and premolar groups, after which group analyses were performed using data obtained from channels 4-13 before and after occlusal adjustment. These results showed that, compared with baseline, brain activity declined significantly in channel 10 of the molar group after adjustment, whereas no significant changes were seen in the premolar group. Intergroup comparison of brain activity in channel 10 revealed a significantly higher level of brain activity in the molar group compared with the premolar and control groups (Fig. 3a), both before and after adjustment. Two-way ANOVA revealed a significant difference $(p<0.05)$ among the molar group and the premolar and control groups.

Meanwhile, intragroup comparison of subjective patient assessments for mastication (i.e., VAS scores) indicated a significantly higher level of discomfort at baseline compared with postadjustment in both the molar and premolar groups, while intergroup comparison failed to reveal any significant differences (Fig. 3b). Two-way ANOVA did not detect any difference between the molar and premolar groups. No significant differences were seen in the control group between the first and second gum chewing.

Moreover, the correlation between PFC activity and VAS score after fitting the restoration was significantly positive only in the molar group (molar group: $r=0.73$,
Fig. 2 Example of patient brain activity before and after adjustment of fitted restoration. This figure shows brain activity data for patient $\mathrm{M} 3$ while chewing gum before and after adjustment of the restoration. Analysis was performed using data from channel 10 (red boxes), indicating brain activity in the medial prefrontal cortex, which is considered to be closely associated with stress response. The green line in each graph represents total hemoglobin (total- $H b$ ), while the red line represents oxygenated hemoglobin $(o x y-H b)$ and the blue line represents deoxygenated hemoglobin (deoxy-Hb)

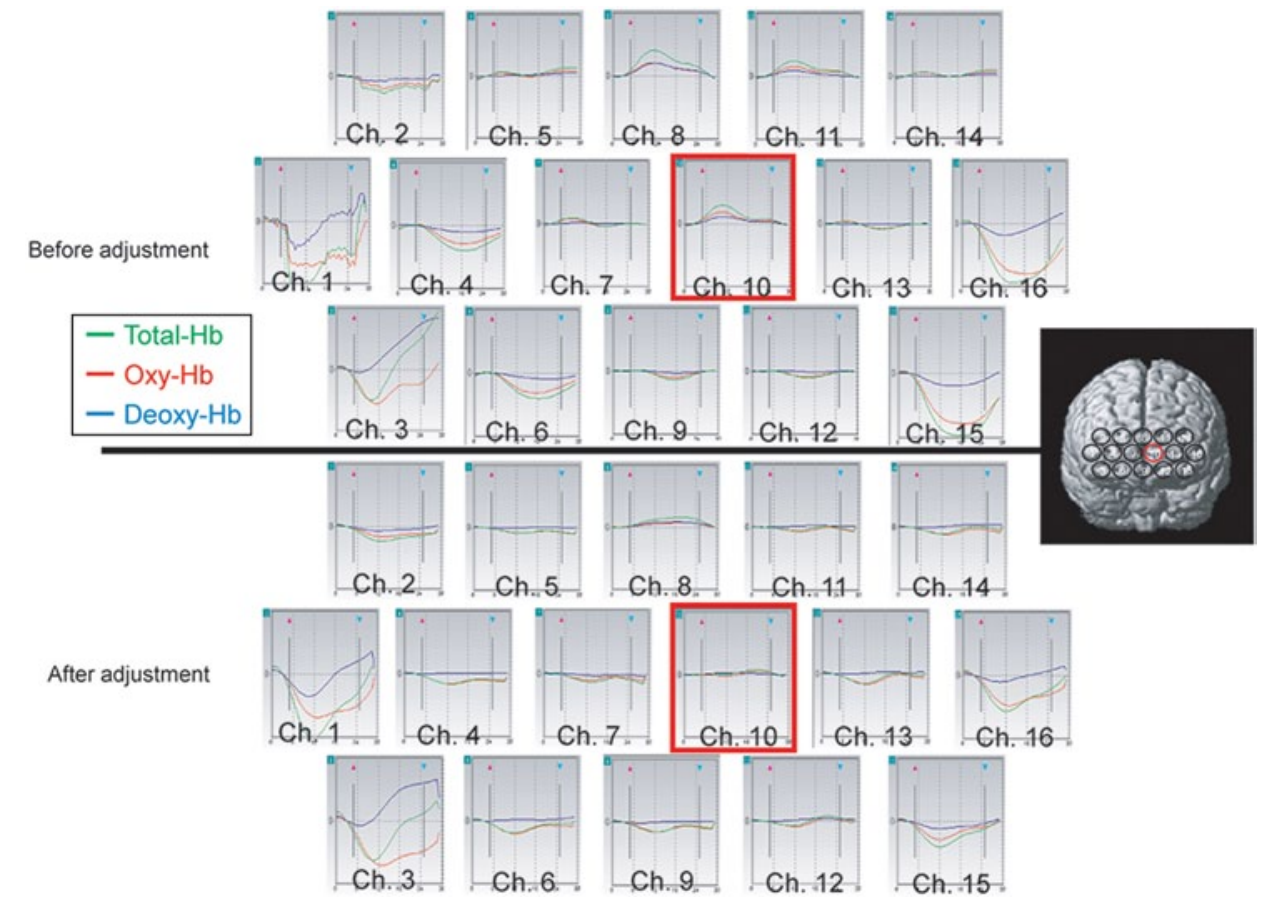




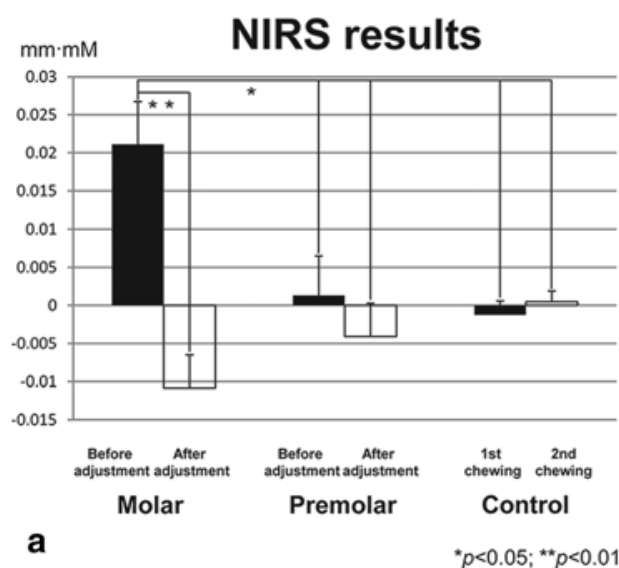

Fig. 3 Brain activity and VAS scores before and after adjustment of fitted restoration. a Results of near-infrared spectroscopy monitoring of medial prefrontal cortex brain activity while chewing gum before and after adjustment or first gum chewing or second gum chewing. b Subjective patient as-

$p<0.01$; premolar group: $r=0.23, p=0.3753$; control group: $r=0.024, p=0.969$ ) (Fig. 4 ).

\section{Discussion}

Modern prosthetic restorations are fabricated, fitted, and adjusted with the aim of restoring occlusal function. However, in most cases, the ultimate goal of occlusal therapy is comfort as determined by the subjective assessment of the patient. The present study therefore sought to gather basic data for the development of an objective chair-side occlusal therapy feedback system. Gum chewing was used to examine the effects that changes in VDO before and after occlusal adjustment of a fitted prosthetic restoration had on both PFC activity and questionnairebased psychological assessments.

All patients were studied at a general dentistry clinic using the OEG-16, which was deemed to be more versatile and portable than other functional neuroimaging systems, in order to assess clinical feasibility. The OEG-
VAS results

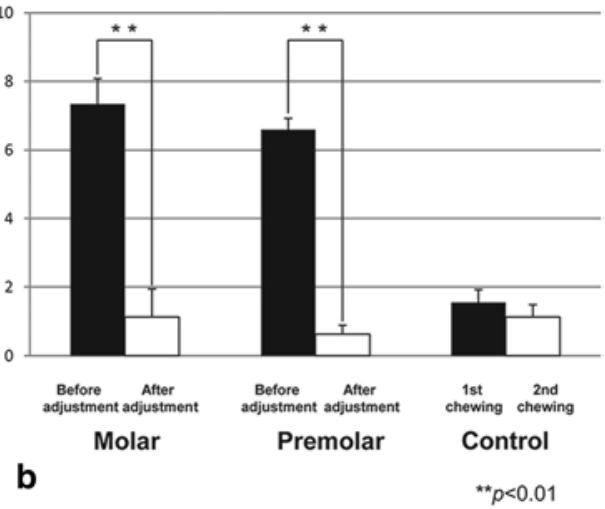

sessment of discomfort while chewing gum before and after adjustment, for the molar group $(n=8)$, premolar group $(n=8)$, and control group $(n=8)$. Values are expressed as mean \pm standard deviation

16 head unit was easy to attach to the PFC and could be worn while the patient chewed gum and when occlusal adjustment was being performed, suggesting that it could be used to develop an instantaneous feedback system capable of real-time assessment of brain activity in clinical settings.

A previous study evaluated changes in global brain activity during clenching using fMRI and discomfort using VAS with an unmodified control splint or a custom-made splint to force the mandible into a retrusive position [6]. As a result, we traced the sensory perception of discomfort associated with retrusion of the mandible to the amygdala and PFC. Located in the cortical region at the rostral end of the frontal lobe, the PFC is responsible for higher cognitive abilities [26] in addition to the perception of stress. When we perceive stress, sensory gating (which is partly mediated by the PFC) plays an important role in filtering effects [27]. Furthermore, the mPFC, located in the frontal pole of the PFC, is believed to be involved in regulating body response to stress by receiving input from the limbic system, including the
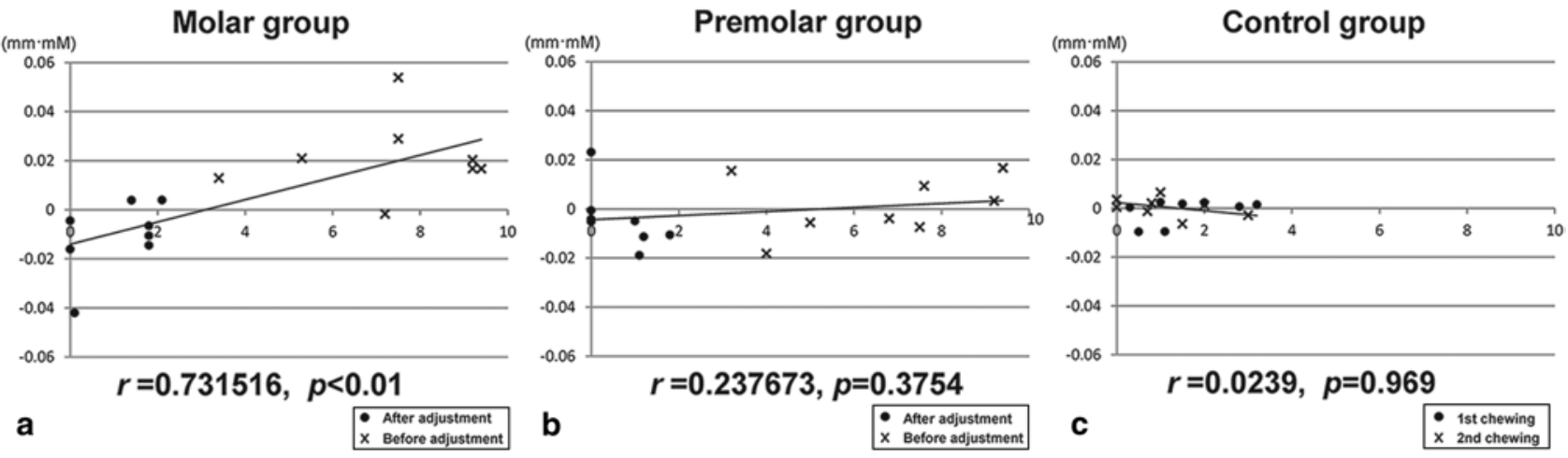

Fig. 4 Correlation between brain activity and VAS score. Scattergrams show correlations between medial prefrontal cortex brain activity and VAS assessment of discomfort in the molar group $(\mathbf{a} ; n=8)$, premolar group $(\mathbf{b} ; n=8)$, and control group (c; $n=8)$ 
hippocampus and amygdala, controlling emotion under stressful conditions, and sending outputs to the brain stem and thalamus [28]. The present study therefore undertook group analyses of brain activity in the PFC using channels $4-13$, based on the hypothesis that chewing discomfort prior to occlusal adjustment may be due to mandibular displacement associated with changes in VDO, and to minimize interference from temporalis muscle activity during gum chewing. The present findings showed a significant decrease in postadjustment brain activity near the MPFC (channel 10) in the molar group, consistent with declines in VAS scores indicating discomfort such as exaggerated perception of restoration height. This suggests that the monitoring of brain activity in the MPFC may be useful for objectively assessing occlusal function in the molar region.

Conversely, no significant increase in postadjustment brain activity was observed in the premolar group, despite similar significant reductions in levels of discomfort compared with the molar group. The control group showed no significant differences in brain activity or discomfort score between the first gum chewing (as a surrogate for preadjustment) and second gum chewing (as a surrogate for postadjustment). The molar group also exhibited a significant positive correlation between brain activity and discomfort $(r=0.73, p<0.01)$.

Oral sensory information is relayed via somatotopically arranged primary afferent fibers originating in the trigeminal ganglion. These fibers terminate in the mandibular nerve dorsal to the principal sensory and spinal trigeminal nuclei, and in the maxillary nerve between the ventral and dorsal aspects of these nuclei. Signals from these nerve fiber terminals then project to the primary somatosensory cortex via the posteromedial ventral nucleus of the thalamus, but localization projects to the PFC via the secondary somatosensory cortex. Stress-related information is assembled within the limbic system (i.e., the hippocampus, amygdala, and PFC) to induce neuroendocrine and behavioral responses [29]. Our results indicate that increased activation of the PFC may have been due to the projection of considerable sensory information on occlusal discomfort from the molars. Meanwhile, the similar feelings of discomfort experienced in both the molar and premolar groups may have been due to the fact that perception of discomfort mainly originates in the limbic system. These findings suggest that monitoring brain activity in the PFC may offer an objective indicator in occlusal treatment of the molar regions, but further research examining global brain activity is needed to identify objective indicators for the premolar regions. Future research should aim to closely monitor brain activity in the PFC, particularly the mPFC, using fMRI or MEG based on tasks similar to those of the present study, and to examine correlations between responses at other brain activity sites, including the limbic system, PFC, and other sites. The sheer diversity of individual occlusal environments may affect the results of brain function monitoring, so consideration of a wide range of factors such as age, sex, maxillofacial morphology, maxillomandibular relationship, and arch form is essential.

The present findings suggest that monitoring PFC activity using NIRS may enable objective assessment of discomfort for occlusal treatment involving the molar regions.

\section{Acknowledgments}

This study was conducted with the approval of the ethics review board at Kanagawa Dental University (Approval no. 158) and was supported by JSPS KAKENHI Grant Numbers 10235286, 24792118.

\section{Conflict of interest}

The authors declare that there are no actual or potential conflicts of interest in relation to this article.

\section{References}

1. Miyachi H, Wake H, Tamaki K, Mitsuhashi A, Ikeda T, Inoue $\mathrm{K}$, Tanaka S, Tanaka K, Miyaoka H. Detecting mental disorders in dental patients with occlusion-related problems. Psychiatry Clin Neurosci. 2007;61:313-19.

2. Woods CD. Self-reported mental illness in a dental school clinic population. J Dent Educ. 2003;67:500-4.

3. Reeves JL 2nd, Merrill RL. Diagnostic and treatment challenges in occlusal dysesthesia. J Calif Dent Assoc. 2007;35:198-207.

4. Otsuka T, Saruta J, Greven M, Ono Y, Sasaguri K, Sato S. Effect of orthodontic reconstruction on brain activity in a patient with masticatory dysfunction. J Stomat Occ Med. 2011;4:76-81.

5. Otsuka T, Watanabe K, Hirano Y, Kubo K, Miyake S, Sato S, Sasaguri K. Effects of mandibular deviation on brain activation during clenching: an fMRI preliminary study. Cranio. 2009;27:88-93.

6. Greven M, Otsuka T, Zutz L, Weber B, Elger C, Sato S. The amount of TMJ displacement correlates with brain activity. Cranio. 2011;29:291-6.

7. Kato K, Ono Y, Kubo KY, Sasaguri K, Watanabe K, Onozuka $M$, Sato $S$. Occlusal disharmony suppresses long-term potentiation in the rat hippocampal CAl region. J Stomat Occ. 2010;3:71-5.

8. Buijs RM, Van Eden CG. The integration of stress by the hypothalamus, amygdala and prefrontal cortex: balance between the autonomic nervous system and the neuroendocrine system. Prog Brain Res. 2000;126:117-32.

9. Gusnard DA, Akbudak E, Shulman GL, Raichle ME. Medial prefrontal cortex and self-referential mental activity: relation to a default mode of brain function. Proc Natl Acad Sci U S A. 2001;98:4259-64.

10. Yasui H, Takamoto K, Hori E, Urakawa S, Nagashima Y, Yada Y, Ono T, Nishijo H. Significant correlation between autonomic nervous activity and cerebral hemodynamics during thermotherapy on the neck. Auton Neurosci. 2010;156:96-103.

11. Momose T, Nishikawa J, Watanabe T, Sasaki Y, Senda M, Kubota K, Sato Y, Funakoshi M, Minakuchi S. Effect of mastication on regional cerebral blood flow in humans examined by positron-emission tomography with ${ }^{15} \mathrm{O}$-labelled water and magnetic resonance imaging. Arch Oral Biol. 1997;42:57-61. 
12. Onozuka M, Fujita M, Watanabe $K$, Hirano $Y$, Niwa M, Nishiyama K, Saito S. Mapping brain region activity during chewing: a functional magnetic resonance imaging study. J Dent Res. 2002;81:743-6.

13. Onozuka M, Fujita M, Watanabe K, Hirano Y, Niwa M, Nishiyama K, Saito S. Age-related changes in brain regional activity during chewing: a functional magnetic resonance imaging study. J Dent Res. 2003;82:657-60.

14. Iida T, Fenwick PB, Ioannides AA. Analysis of brain activity immediately before conscious teeth clenching using magnetoencephalographic method. J Oral Rehabil. 2002;34:487-96.

15. Narita N, Kamiya K, Yamamura K, Kawasaki S, Matsumoto T, Tanaka N. Chewing-related prefrontal cortex activation while wearing partial denture prosthesis: pilot study. J Prosthodont Res. 2009;53:126-35.

16. Takeda T, Shibusawa M, Sudal O, Nakajima K, Ishigami $\mathrm{K}$, Sakatani K. Activity in the premotor area related to bite force control-a functional near-infrared spectroscopy study. Adv Exp Med Biol. 2010;662:479-84.

17. Shibusawa M, Takeda T, Nakajima K, Jun H, Sekiguchi S, Ishigami K, Sakatani K. Functional near-infrared spectroscopy study on primary motor and somatosensory cortex response to biting and finger clenching. Adv Exp Med Biol. 2010;662:485-90.

18. Iida T, Kato M, Komiyama O, Suzuki H, Asano T, Kuroki T, Kaneda T, Svensson P, Kawara M. Comparison of cerebral activity during teeth clenching and fist clenching: a functional magnetic resonance imaging study. Eur J Oral Sci. 2010;118:635-41.

19. Iida T, Sakayanagi M, Svensson P, Komiyama O, Hirayama T, Kaneda T, Sakatani K, Kawara M. Influence of periodontal afferent inputs for human cerebral blood oxygenation during jaw movements. Exp Brain Res. 2012;216:375-84.

20. Shimazaki T, Otsuka T, Akimoto S, Kubo KY, Sato S, Sasaguri K. Comparison of brain activation via tooth stimulation. J Dent Res. 2012;91:759-63.
21. Watanabe E, Yamashita Y, Maki A, Ito Y, Koizumi H. Noninvasive functional mapping with multi-channel near infra-red spectroscopic topography in humans. Neurosci Lett. 1996;205:41-4.

22. Okamoto M, Dan H, Sakamoto K, Takeo K, Shimizu K, Kohno S, Oda I, Isobe S, Suzuki T, Kohyama K, Dan I. Three-dimensional probabilistic anatomical cranio-cerebral correlation via the international 10-20 system oriented for transcranial functional brain mapping. Neuroimage. 2004;21:99-111.

23. Throckmorton GS, Buschang BH, Hayasaki H, Phelan T The effects of chewing rates on mandibular kinematics. J Oral Rehabil. 2001;28:328-34.

24. Hoshi Y, Kobayashi N, Tamura M. Interpretation of nearinfrared spectroscopy signals: a study with a newly developed perfused rat brain model. J Appl Physiol. 2001;90:1657-62.

25. Yamamoto T, Kato T. Paradoxical correlation between signal in functional magnetic resonance imaging and deoxygenated hemoglobin content in capillaries: a new theoretical explanation. Phys Med Biol. 2002;47:1121-41.

26. McPherson S, Cummings JL. The frontal lobes and frontalsubcortical circuits in neuropsychiatric disorders. In: Grafman J, Editor. Handbook of neuropsychology. vol. 7, 2. Ed. Amsterdam: Elsevier; 2002. pp. 99-116.

27. Karl A, Malta LS, Maercker A. Meta-analytic review of event-related potential studies in post-traumatic stress disorder. Biol Psychol. 2006;71:123-47.

28. Bandler R, Keay KA, Floyd N, Price J. Central circuits mediating patterned autonomic activity during active vs. passive emotional coping. Brain Res Bull. 2000;53:95-104.

29. Fuchs E, Flügge G. Chronic social stress: effects on limbic brain structures. Physiol Behav. 2003;79:417-27. 